International Journal of Advanced Technology in Mechanical, Mechatronics and Materials

(IJATEC)

Vol. 01, No. 2 (2020) 50-55

(C) Institute for Research on Innovation and Industrial System (IRIS)

\title{
The Effect of Ball Size on Hardness of Mechanically Alloyed Al-10wt.\%Ti Powders
}

\author{
Adolf Asih Supriyanto*a, Widodo ${ }^{\text {, }}$, Ade Irvan Tauvana ${ }^{\mathrm{b}}$, Syafrizal $^{\mathrm{b}}$ \\ ${ }^{a}$ Mechatronics Engineering, Politeknik Enjinering Indorama, Purwakarta, Indonesia \\ ${ }^{b}$ Mechanical Engineering, Politeknik Enjinering Indorama, Purwakarta, Indonesia
}

\begin{abstract}
Particle size and hardness of Al-10\%wt.Ti powders due to the influence of ball diameter have been carried out using mechanical alloying techniques. The purpose of this study was to determine the effect of ball size on the hardness of Al-10\%wt.Ti powders. The milling device used consisted of a Fritsch Pulverisette-5 planetary type ball mill with the rotational speed of about $360 \mathrm{rpm}$ and balls size of 10,15 and $20 \mathrm{~mm}$. The materials used are stearic acid which was used as a process control agent, aluminum, and titanium powders. A mixture of aluminum and titanium powders has the composition of Al-10wt.\%Ti. The Al-10wt.\% Ti powders, stearic acid and stainless-steel balls were added to the Fritsch Pulverisette-5 planetary in the argon gas environment. The weight ratio of stainless steel balls to the Al-10wt.\% Ti powder was $20: 1$. The mixing time process was carried out for 5, 10, 15, 20 and 30 hours, respectively. Changes in phase compositions of these Al-Ti powders under different ball mill sizes were examined by XRD, and the optimum experimental parameter was obtained: the ball mill size was $20 \mathrm{~mm}$. It was found that from the diffraction patterns of the Al-10wt.\%Ti powders, the peaks of titanium begin to disappear with the increasing of the milling time, which indicates the increasing degrees of alloying of titanium atoms in the aluminum matrix. The microhardness test results showed that the hardness increases with increasing ball size are about $10.7 \%$ when milled at 5 hours and $17.8 \%$ when milled at 30 hours.
\end{abstract}

Keywords: ball size; particle size; hardness

DOI: $10.37869 /$ ijatec.v1i2.21

Received 8 June 2020; Accepted 31 July 2020; Available online 7 August 2020

(C) 2020 IRIS Publisher. All rights reserved.

\section{Introduction}

TiAl intermetallic alloys have been extensively investigated over the past two decades. Nowadays a major challenge in the applications of TiAl intermetallics comes from their high cost and production difficulties [1-4]. Mostly ingot metallurgy is used to produce TiAl based alloys. However, the TiAl alloys produced in this way generally have very coarse grain. Alternatively, the AlTi alloy can be prepared by powder metallurgy, particularly by mechanical alloying, which readily ensures structural refinement and chemical homogeneity [5-8].

$\mathrm{Al}-\mathrm{Ti}$ alloys have been one of the materials used for aerospace structural and engine application, because they have high melting points and lower densities together with high strength $[3,7,9]$. In this paper, it is reported the used of mechanical alloying process and stearic acid as a PCA to prepare the hardness of Al-Ti alloy due to the influence of ball diameter.

\section{Research Methodology}

The starting materials are fine powders of $\mathrm{Al}$ and $\mathrm{Ti}$ were mixed to prepare the sample of $\mathrm{Al}$ 10wt.\%Ti. The Al and Ti powders used had a mean particle size of about 71 and $60 \mu \mathrm{m}$, respectively. 
A Fritsch Pulverisette-5 planetary type ball mill with a rotational rate of $360 \mathrm{rpm}$ and the ball to powder weight ratio was about $20: 1$ was used for the mechanical alloying of the sample. The sample mixture was mixed with stainless steel balls and sealed under argon atmosphere in a cylindrical stainless-steel container. Stearic acid $\left(\mathrm{CH}_{3}-\left(\mathrm{CH}_{2}\right)_{16}-\mathrm{COOH}\right)$ of about $4 \mathrm{wt} . \%$ of the sample mixture was used as a PCA. The balls sizes were 10,15 and $20 \mathrm{~mm}$. Several milling times were used ranging 2 up to 30 hours, interrupted for 30 minutes every 2.5 hours in order to minimize excessive temperature rise and to limit adherence of the powder to container walls $[6,10]$.

Samples after milling were characterized by X-ray diffraction (XRD) method (in a Siemen D5000 $\mathrm{X}$-ray diffractometer) with $\mathrm{Cu} \mathrm{K}_{\alpha}$ radiation $(\lambda=0.15406 \mathrm{~nm})$. The XRD patterns were recorded in the $2 \theta$ range between 20 and $80^{\circ}$ with steps of $0.025^{\circ}$.

The resulting powder is pelleted (green body) $1 \mathrm{~cm}$ in diameter by applying a pressure of 10 tons. The pellets were then characterized by X-ray diffraction (XRD) technique, scanning electron microscopy (SEM) and microhardness (Vickers) with a load of $100 \mathrm{gf}$.

\section{Results and Discussions}

Figure 1 shows the X-ray diffraction patterns of Al-10wt.\%Ti powder milled for 30 hours with different ball sizes and stearic acid as a PCA. From the pattern, it can be seen that the Ti peaks still visible when the ball sizes is $10 \mathrm{~mm}$, whereas they disappear at a high ball sizes of $20 \mathrm{~mm}$, which indicates the enhancement of the mechanical alloying process with the increasing of the ball sizes.

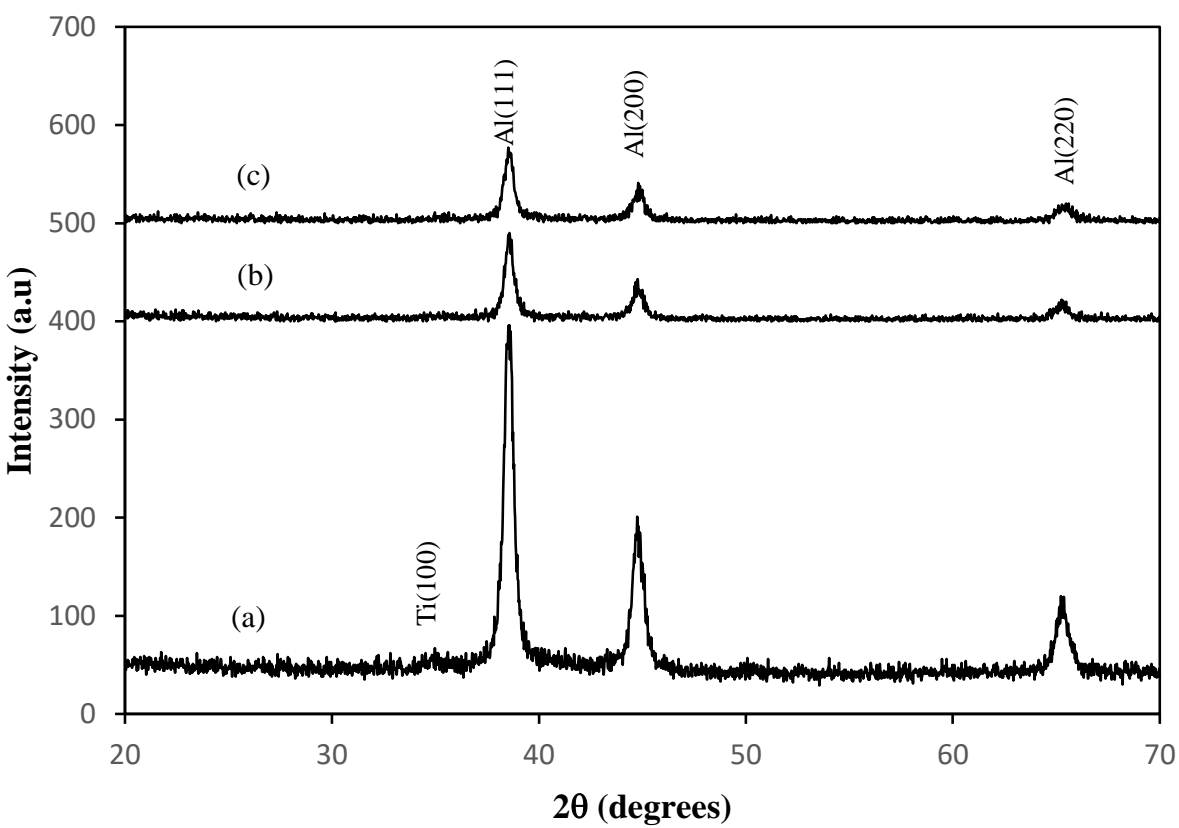

Figure 1. XRD patterns of Al-10wt.\%Ti powders milled for 30 hours with different ball sizes: a) 10, b) 15 and c) $20 \mathrm{~mm}$

Enlarged fragments for the diffraction patterns of Al-10wt.\%Ti powder milled after 30 hours with different ball size in the range from $37^{\circ}$ to $40^{\circ}$ is shown in Figure 2. From the pattern, the peak position of Al reflections shifts to higher $2 \theta$ angles. The peak position of the $\mathrm{Al}$ reflection for the ball sizes of 10,15 , and $20 \mathrm{~mm}$ are $38.5400^{\circ}, 38.5626^{\circ}$, and $38.5645^{\circ}$ respectively, relating to the reduction of the $\mathrm{Al}$ lattice parameter, due to Ti dissolution in $\mathrm{Al} \mathrm{[10].} \mathrm{The} \mathrm{Al}$ lattice parameter, as calculated from the XRD pattern, is shown in Figure 3. The decrease of the Al lattice parameter indicates that atoms of $\mathrm{Ti}$ occupy some positions in the bcc lattice of Al during the process of mechanical alloying, causing the $\mathrm{Al}$ lattice to shrink. This suggests that the alloying between $\mathrm{Al}$ and Ti mainly takes place at 30 hours milling and the formation of $\mathrm{Al}(\mathrm{Ti})$ solid solution has completed at about 30 hours with ball size $20 \mathrm{~mm}$.

From Figure 2, the broadening of the $\mathrm{Al}$ peaks is commonly attributed to the structural change in the samples, such as the formation of amorphous phases, large lattice strains in grains, or the 
embedding of very small crystals in an amorphous matrix [10]. Using the Hall-Williamson Method, the crystallite size of the Al-10wt\%Ti powders after mechanical alloying for various ball sizes is shown in Figure 4. The crystallite size of the powders decreases from the initial sizes to about 15.7 $\mathrm{nm}$ after 30 hours milling with ball size $20 \mathrm{~mm}$.

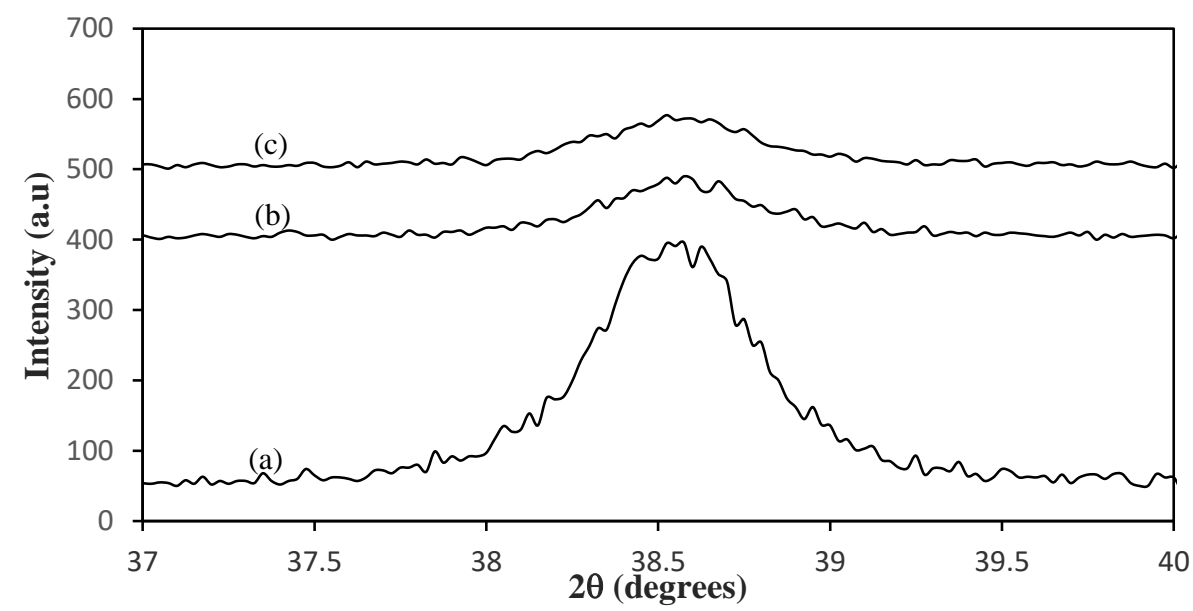

Figure 2. Enlarged fragments of the diffraction patterns in the range from 37 to $40^{\circ}$, for different ball sizes: a) 10, b) 15 and c) $20 \mathrm{~mm}$

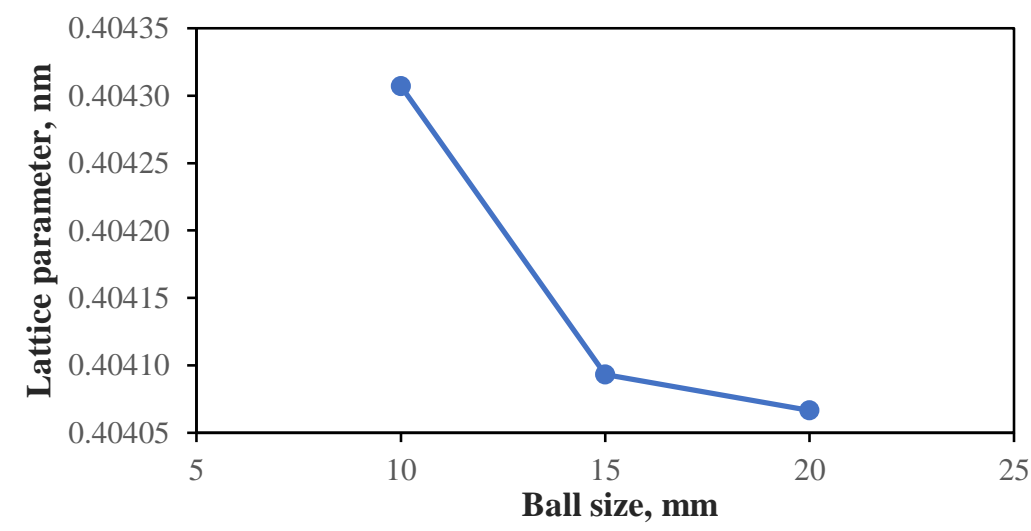

Figure 3. Lattice parameter as a function of ball sizes

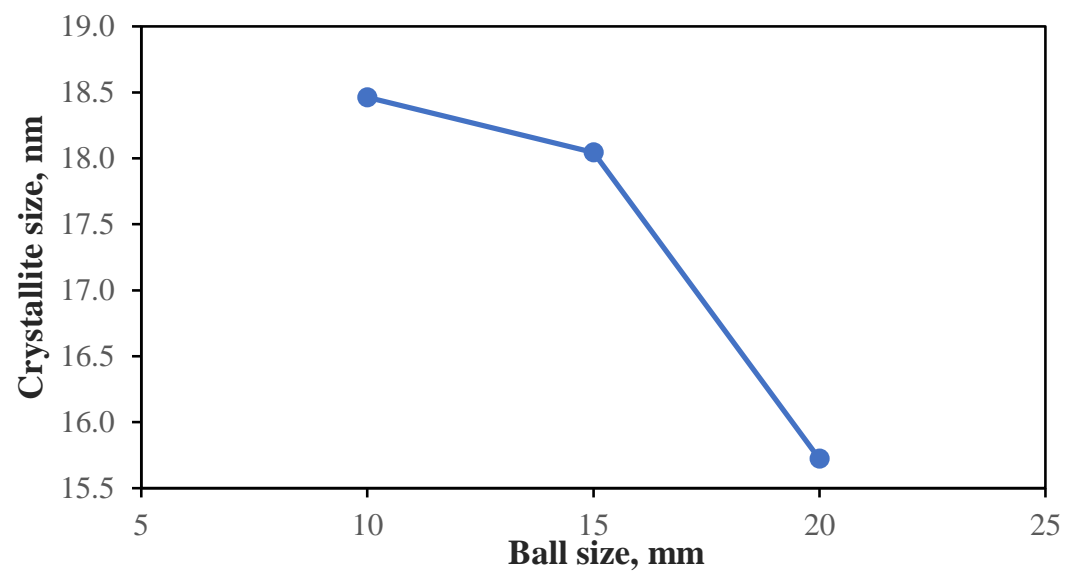

Figure 4. The crystallite size of the Al-10wt\%Ti powders as a function of ball sizes

Figure 5 is the X-ray diffraction patterns of $\mathrm{Al}-10 \mathrm{wt} . \% \mathrm{Ti}$ powder milled after different milling times. The ball-to-powder ratio, the balls mill sizes and a rotational rate were fixed at 20:1, $20 \mathrm{~mm}$, and $360 \mathrm{rpm}$ respectively. At the beginning, the peaks from both aluminum and titanium are clearly seen. All the peaks at $2 \theta=35.09^{\circ}, 38.52^{\circ}$ and $40.32^{\circ}$ are attributed to (100), (002) and (101) crystal planes. These $2 \theta$ values could be well indexed to titanium with hexagonal crystal structure (JCPDS no. 44-1294) and the lattice parameters are $a$ and $b=2.9500 \AA$ and $c=4.683 \AA$. While the peak at $2 \theta$ 
$=38.52^{\circ}, 44.75^{\circ}, 65.16^{\circ}$ and $78.29^{\circ}$, corresponding to (111), (200), (220) and (311) crystal planes, are indexing to aluminum with cubic crystal structure (JCPDS no. 04-0787) and the lattice parameters are $a, b$ and $c=4.04940 \AA ̊$. No impurities were detected.

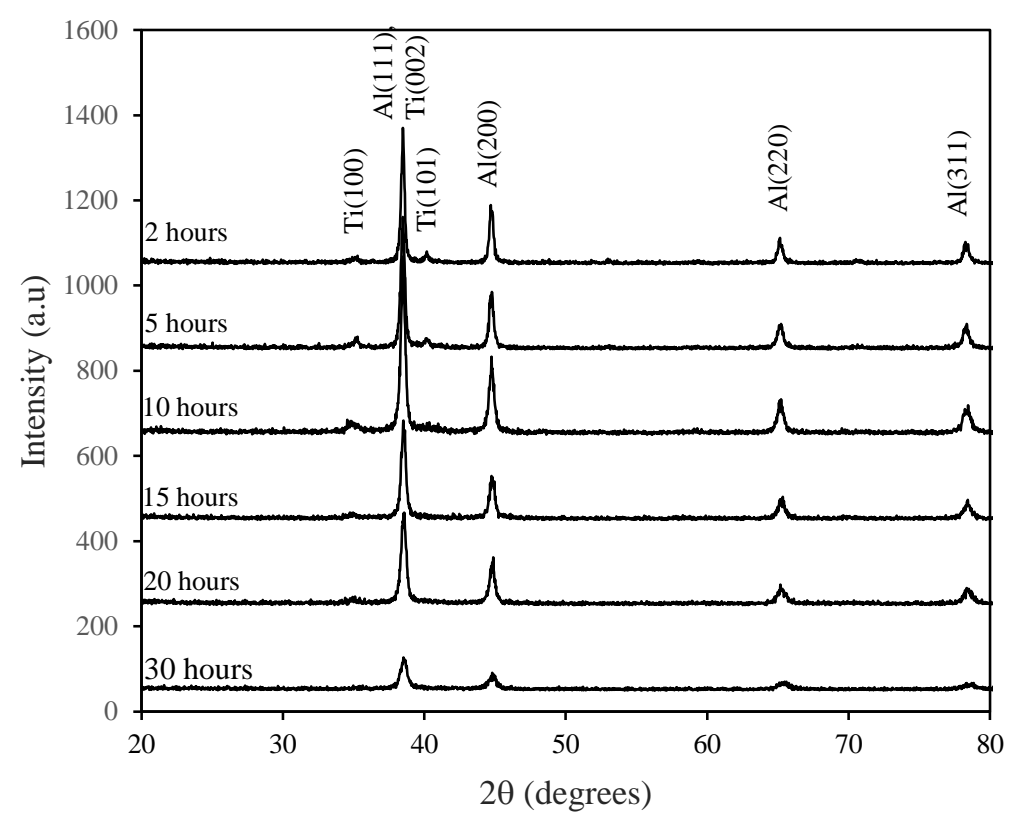

Figure 5. The crystallite size of the Al-10wt\%Ti powders as a function of ball sizes

It can be seen that the Ti peaks are still visible when the milling time is 15 hours (Figure 5). But with the increasing milling time at 20 hours, the Ti peaks tend to disappear which indicates the alloying of $\mathrm{Al}$ and Ti elements [10]. When the milling time continues to increase and reaches 30 hours, all the Ti peaks disappear, revealing the completion of the alloying. These phenomena can be showed that Ti diffuses into the bcc structures and form alloying of Ti atoms in the Al matrix.

Figure 6 shows the SEM micrograph of Al-10wt\%Ti powders after milling at 30 hours with the ball size of 15 and $20 \mathrm{~mm}$. At the beginning, the aluminum and titanium powders were combined to form sheets or laminates. When the milling time continues to increase and reaches 30 hours, it is found that the laminate forms into small clusters near the spherical shape due to repeated fracture and cold welding (Figure 6). The particle size of Al-10wt\%Ti powders milled at 30 hours with ball size of $20 \mathrm{~mm}$ is smaller than that one with ball size of $15 \mathrm{~mm}$. From Figure 6, the average particle size of Al-10wt\%Ti powders milled with ball size of $20 \mathrm{~mm}$ at 30 hours is about $14 \mathrm{~mm}$ and that one with ball size $15 \mathrm{~mm}$ is about $21 \mathrm{~mm}$. It agrees with the XRD result.
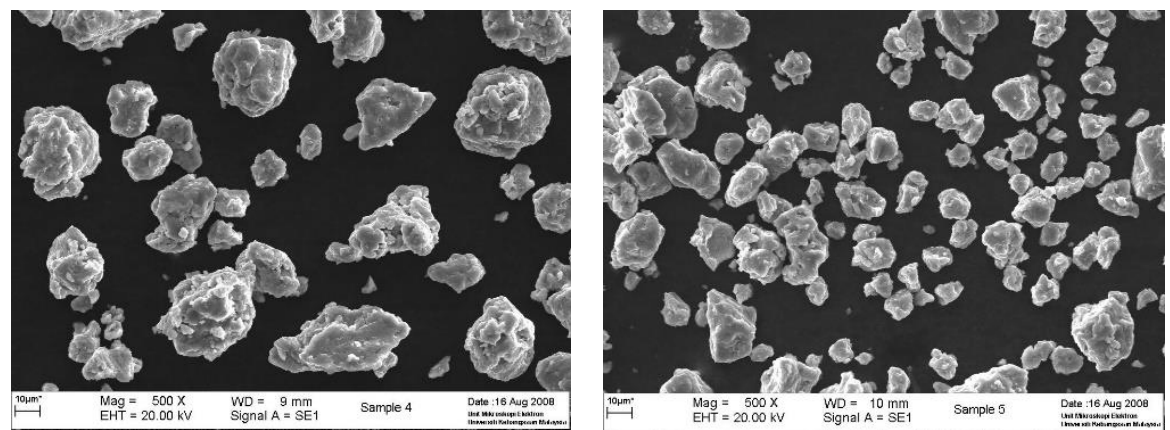

Figure 6. Al-10wt\%Ti powders milled for 30 hours with diameter: a) 15 and b) $20 \mathrm{~mm}$

Figure 7 shows the influence of milling time and ball size on the hardness of Al-10wt.\%Ti alloy pellets. The hardness of Al-10wt.\%Ti alloy pellets increases with increasing milling time and ball size. This phenomenon is due to the more milling time, the finer the Al-Ti alloy particles are produced then the pellets get harder. The hardness of Al-10wt\%Ti powders milled at 5 hours with ball size of $20 \mathrm{~mm}$ is harder than that one with ball size of $15 \mathrm{~mm}$. Average hardness of Al-10wt\%Ti powders milled with ball size of $20 \mathrm{~mm}$ at 5 hours is $279 \mathrm{Hv}$, while that one with ball size $15 \mathrm{~mm}$ is $252 \mathrm{Hv}$. Meanwhile, 
the hardness of Al-10wt\%Ti powders milled at 30 hours with ball size of $20 \mathrm{~mm}$ is $606 \mathrm{Hv}$, and that one with ball size of $15 \mathrm{~mm}$ is $514 \mathrm{Hv}$. The hardness increment from Al-10wt\%Ti powders milled with different ball size (15 dan $20 \mathrm{~mm}$ ) at 5 and 30 hours are about 10.7 and $17.8 \%$ respectively.

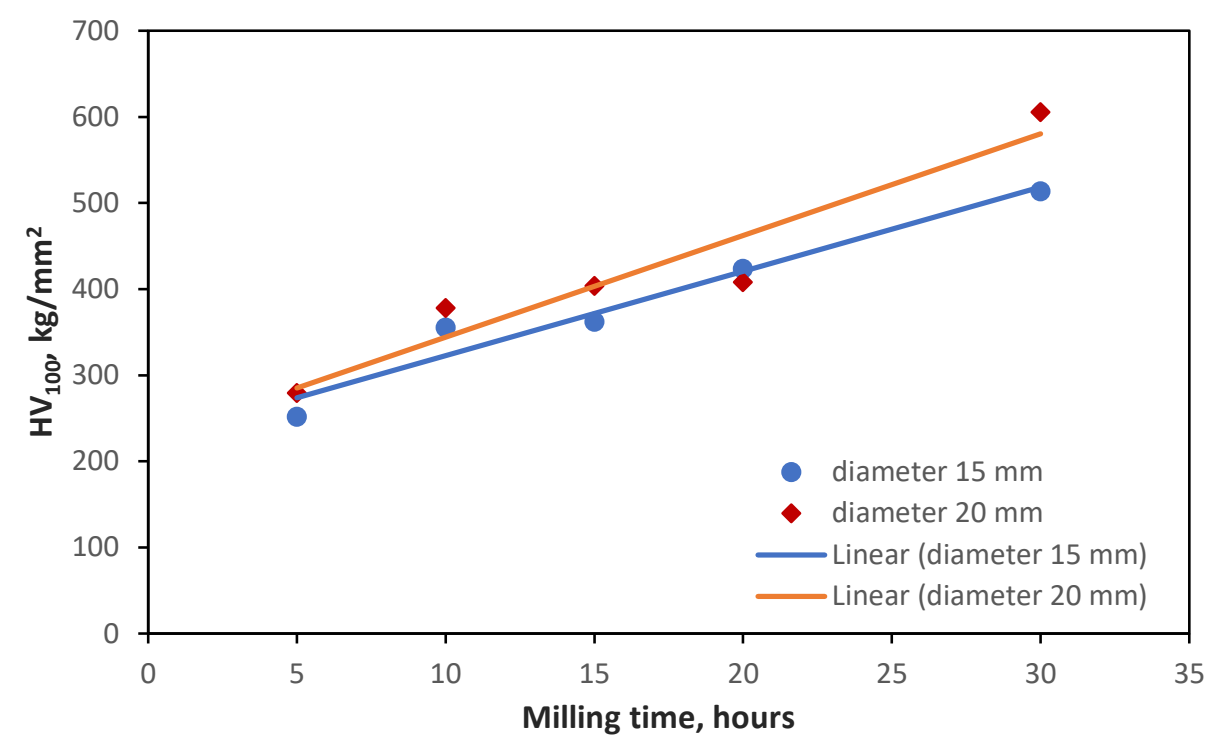

Figure 7. The influence of milling time and ball size on the hardness of Al-10wt.\%Ti alloy pellets

\section{Conclusions}

The alloying degree is improved with the increasing of milling time during the mechanical alloying process of the Al-Ti alloy. It reveals that from the diffraction patterns of the Al-10wt.\%Ti powders, the peaks of titanium begin to disappear with the increasing of the milling time, which indicates the increasing degrees of alloying of titanium atoms in the aluminum matrix. The Ti peaks disappear at a high ball sizes of $20 \mathrm{~mm}$, which indicates the enhancement of the mechanical alloying process with the increasing of the ball sizes. The hardness increases increase with increasing milling time and ball size. The hardness increment from Al-10wt\%Ti powders milled with different ball size (15 dan 20 $\mathrm{mm})$ at 5 and 30 hours are about 10.7 and $17.8 \%$ respectively.

\section{Acknowledgments}

We would like to thank to the School of Applied Physics, FST, UKM for the providing laboratory facility and to En. Aswani and En. Razali for their technical assistance.

\section{References}

[1] H. R. Khan and M. A. Siddiqui. Effect of Milling Time on Al-Fe-Cr-20 Wt . \% $\mathrm{Al}_{2} \mathrm{O}_{3}$ composite Prepared Through Ball Milling. J. Eng. Res. Appl., 2014;4(7):183-188.

[2] A. H. Seikh, M. Baig, and H. R. Ammar. Corrosion behavior of nanostructure al-fe alloy processed by mechanical alloying and high frequency induction heat sintering. Int. J. Electrochem. Sci., 2015;10(4):3054-3064

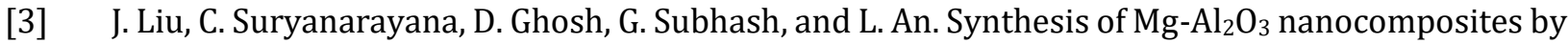
mechanical alloying. J. Alloys Compd., 2013;563:165-170.

[4] M. Khaloobagheri and S. A. Barfjan. The Effect of Milling Time on Properties and Microstructure of Cu-Yttria Stabilized Zirconia Composites Fabricated by Powder Metallurgy. 2015;1(2):78-84.

[5] X. W. Li, H. F. Sun, W. Bin Fang, and Y. F. Ding. Structure and morphology of Ti-Al composite powders treated by mechanical alloying. Trans. Nonferrous Met. Soc. China, 2011; 21(2): s338s341.

[6] N. Forouzanmehr, F. Karimzadeh, and M. H. Enayati. Study on solid-state reactions of nanocrystalline TiAl synthesized by mechanical alloying. J. Alloys Compd., 2009:471(1-2): 93-97.

[7] B. Gabbitas, P. Cao, S. Raynova, and D. Zhang. Microstructural evolution during mechanical milling of Ti/Al powder mixture and production of intermetallic TiAl cathode target. J. Mater. Sci., 2012;47(3): 1234-1243. 
[8] M. R. Farhang, A. R. Kamali, and M. Nazarian-Samani. Effects of mechanical alloying on the characteristics of a nanocrystalline Ti-50 at.\%Al during hot pressing consolidation. Mater. Sci. Eng. B Solid-State Mater. Adv. Technol., 2010;168(1): 136-141.

[9] J. B. Al-Dabbagh, R. M. Tahar, S. A. Harun, and M. Ishak. Structural and phase formation of TiAl alloys synthesized by mechanical alloying and heat treatment. Int. J. Nanoelectron. Mater., 2015; 8(1):23-32.

[10] A. A. Supriyanto and A. R. Daud. Effect of milling time on microstructure of mechanically alloyed Al-Ti powders. AIP Conf. Proc., 2009; 1202: 117-121. 